\title{
Impacts De L'urbanisation Sur Les Caractéristiques Morphologiques Et Les Apports En Eau Du Barrage Hydro-Agricole De Nanan Dans Le Département De Yamoussoukro (Côte d'Ivoire)
}

\author{
Sawadogo Zounabo Épouse Kouyate, \\ Institut National Polytechnique Houphouët Boigny de Yamoussoukro, \\ UMRI Science Agronomique et Génie Rural et \\ Université Péleforo Gbon Coulibaly de Korhogo, Côte d'Ivoire \\ Yao Casimir Brou,
} Institut National Polytechnique Houphouët Boigny de Yamoussoukro, UMRI Science Agronomique et Génie Rural, Côte d'Ivoire

Gneneyougo Emile Soro, Bi Tié Albert Goula, Université Nangui Abrogoua d'Abidjan, Côte d'Ivoire Laboratoire de Géosciences et de l'Environnement

Doi: 10.19044/esj.2018.v14n12p89 URL:http://dx.doi.org/10.19044/esj.2018.v14n12p89

\begin{abstract}
The significant decrease in the water level of the Nanan agricultural dam is becoming very worrying for rice farmers. In this study, we analyzed the land use dynamics of dam watershed and its impacts on morphological characteristics and reservoir inflow. This analysis was conducted by coupling the satellites images of Landsat sensors over a thirty years, Digital Terrain Model (DTM) and direct field observation. The classification of Thematic Mapper (1986), Enhanced Thematic Mapper+ (2000) and Operational Land Imager (2015) images has gave respectively an overalls accuracies of $99.01 \%$, $96.62 \%$ and $94.6 \%$ and kappa index of $0.98,0.95$ and 0.91 . The analysis of the land use dynamics of the catchment showed that its urbanization started before 1986 and accelerated after 2000 with the transfer of the political capital of Abidjan to Yamoussoukro. The area of dam watershed was reduced between $1986\left(8,272 \mathrm{~km}^{2}\right)$ and $2000\left(7,348 \mathrm{~km}^{2}\right)$.After 2000, the construction of road infrastructures on the watershed led to a subdivision of the watershed into two sub-watersheds separated by a culvert. The current configuration is an upstream basin with an area of $4.30 \mathrm{~km}^{2}$ and the downstream basin an area of $3.048 \mathrm{~km}^{2}$. As a hydrological consequence of urbanization in watershed is runoff water has increased from $41.7 \mathrm{~mm}$ in 1986 to $43.30 \mathrm{~mm}$ in 2000 and
\end{abstract}


$46.40 \mathrm{~mm}$ in 2015. However, inter-basin water transfer and grass cover at the entrance to the basin slowed runoff and reduced by a third the flow to the reservoir dam. This situation led to a decrease in the water level in the reservoir of the Nanan hydro-agricultural dam.

Keywords: Agricultural dam, catchment, Remote sensing, Yamoussoukro, Côte d'Ivoire

\section{Resume}

La réduction considérable du niveau du plan d'eau du barrage agricole de Nanan devient de plus en plus préoccupante pour les riziculteurs. Dans cette étude, nous avons analysé l'évolution spatio-temporelle du mode d'occupation $\mathrm{du}$ sol sur le bassin versant du barrage de Nanan et ses incidences sur les caractéristiques morphologiques et les apports en eau. Cette analyse a été menée grâce au couplage des images satellitaires des capteurs Landsat sur une période de 30 ans, de Modèle Numérique de Terrain (MNT) et d'observation directe de terrain. La classification des images Thematic Mapper (1986), Enhanced Thematic Mapper+ (2000) et Operational Land Imager (2015) a donné respectivement une précision globale de $99,01 \%, 96,62 \%$ et $94,6 \%$ et un indice Kappa de 0.98 , 0,95 et 0,91. L'analyse de l'évolution spatiotemporelle du mode d'occupation du sol du bassin versant a montré que son urbanisation date d'avant 1986 et s'est accélérée après les années 2000 suite au démarrage des travaux du transfert de la capitale politique d'Abidjan à Yamoussoukro. La surface topographique du bassin versant estimée en 1986 à $8,272 \mathrm{~km}^{2}$ est passée à $7,348 \mathrm{~km}^{2}$ en 2000 . Après 2000 , la réalisation d'infrastructures routières sur le bassin versant a entrainé une subdivision de celui-ci en deux sous bassins versants séparés par un dalot. La configuration actuelle de la zone d'étude est un bassin amont à une superficie de 4,30 $\mathrm{km}^{2}$ et le bassin aval, une superficie de $3,048 \mathrm{~km}^{2}$. Comme conséquence hydrologique de ces différentes modifications morphologiques du bassin versant, la lame d'eau ruisselée est passée de 41,7 mm en 1986 à 43,30 mm en 2000 et $46,40 \mathrm{~mm}$ en 2015. Cependant, le transfert d'eau inter-bassins et l'enherbement de l'entrée de la cuvette ont ralenti les ruissellements et réduit de tiers les écoulements vers la retenue du barrage. Ce qui a entrainé une baisse du niveau de l'eau dans la retenue du barrage hydro-agricole de Nanan.

Mots clés : Barrage hydro-agricole, bassin versant, télédétection, Yamoussoukro, Côte d'Ivoire

\section{Introduction}

Pour accroître les productions agropastorales et assurer un développement socio-économique des populations, la Côte d'Ivoire, s'est 
engagée depuis 1970, dans la maîtrise des ressources en eau par la réalisation de 592 petites retenues d'eau à vocation agricole et pastorale au Centre et au Nord (Cecchi et al., 2007). Face aux menaces du changement climatique et de pressions anthropiques sur les différents écosystèmes, plusieurs auteurs se sont intéressés à l'impact de ces phénomènes sur les grands fleuves et barrages (Kouakou et al., 2007, Soro et al., 2011, Yao et al., 2012). Cependant, les études sur les petits barrages notamment à vocation agricole sont rares. Pourtant, ces petites retenues permettent d'assurer la sécurité alimentaire, de lutter contre la pauvreté et l'exode rural et de garantir la cohésion sociale (Fowe et al., 2015). Les producteurs ainsi que les populations vivant dans les zones urbaines à proximité des retenues profitent de ces retombés à travers la disponibilité des denrées alimentaires et le développement de commerce interne. Le barrage de Nanan dans le département de Yamoussoukro, choisi pour la présente étude est représentatif de ces petits barrages agricoles périurbains du centre de la Côte d'Ivoire.

En effet, ces dernières années, les riziculteurs du périmètre de Nanan n'arrivent pas à réaliser deux cycles culturaux comme par le passé et la superficie emblavée a diminué. Sur les 32 hectares réhabilités en 2001 pour la riziculture par le Projet Riz Centre, seulement 16 hectares sont exploités effectivement. L'une des raisons évoquées par les riziculteurs pour expliquer cette situation, est la baisse de la pluviométrie ayant entrainé une baisse considérable du niveau d'eau du barrage. Cette observation avait déjà été faites dans plusieurs études (Ardoin et al., 2003 ; Bigot et al., 2005 ; Goula et al., 2006 et Guy et al., 2015). Cependant, une reprise pluviométrique après les épisodes secs des années 1970 a été annoncée dans plusieurs zones de la Côte d'Ivoire (Goula et al., 2006 ; Kouassi, 2007) et même à Yamoussoukro à partir des années 1992 (N'Guessan et al., 2013). Paradoxalement à cette reprise, le niveau d'eau dans le barrage de Nanan est toujours en baisse. Il est alors nécessaire dans le cadre de la compréhension de la baisse du niveau d'eau du barrage de Nanan, d'analyser des facteurs autre que la pluviométrie susceptibles d'être à l'origine de cet état de fait.

Par ailleurs, la ville de Yamoussoukro à l'image de la Côte d'Ivoire connaît un fort taux de croissance démographique annuel (2,26\% de 1998 2014) avec un taux d'urbanisation actuel de l'ordre de 66,9\% (RGPH, 2014), faisant de celle-ci, une ville en pleine mutation. Or, les changements les plus rapides et les plus importants se produisent dans les zones périurbaines des grandes agglomérations. Et les bassins versants associés à ces transformations, voient le régime hydro-géomorphologique de leurs cours d'eau affecté par celles-ci (Braud, 2011). Selon leur nature, les hétérogénéités d'un bassin versant peuvent faire office d'obstacles aux écoulements (haies derrières lesquelles le ruissellement va s'accumuler, bassins d'infiltration...) (Braud, 2011). En outre, l'urbanisation en cours dans la zone d'étude, n'aurait-elle pas 
affectée la morphologie et l'hydrologie du bassin versant périurbain du barrage de Nanan?

L'objectif de cette étude est d'analyser la dynamique spatio-temporelle de l'occupation du sol du bassin versant de Nanan et l'impact de l'urbanisation sur ses caractéristiques morphologiques et ses apports en eau.

\section{Materiel et methodes}

\section{Présentation de la zone d'étude}

La zone d'étude est située en périphérie sud de la circonscription administrative de Yamoussoukro, au centre de la Côte d'Ivoire, à environ 230 $\mathrm{km}$ au Nord d'Abidjan (figure 1).

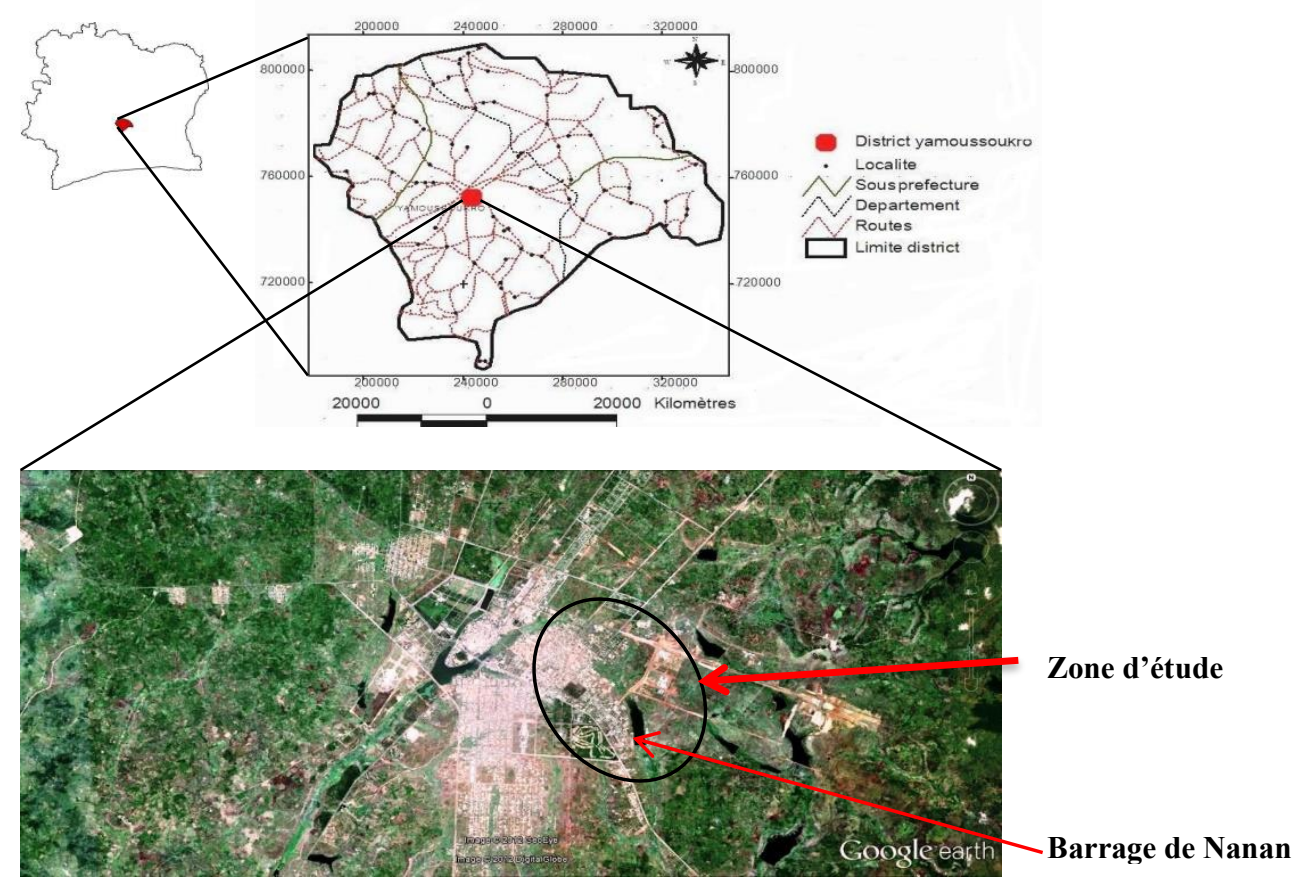

Figure 1 : Localisation de la zone d'étude (Google earth, Janvier 2016)

Il est situé entre les parallèles $6^{\circ} 46.1^{\prime}$ 'et $6^{\circ} 49.7^{\prime}$ de latitude Nord, et les méridiens $5^{\circ} 13.64^{\prime}$ et $5^{\circ} 15.44^{\prime}$ de longitude Ouest. Avec une superficie de $8 \mathrm{~km}^{2}$, il englobe une partie des quartiers « Millionnaire », «Kpankpassou » et « N'Zuessy ». Le barrage de Nanan a été construit en 1970 sur un affluent en rive droite de la rivière « KPOUSSOU », lui-même affluent en rive gauche du fleuve Bandama, plus précisément le Bandama rouge (la Marahoué) (BNETD, 1997). La zone d'étude appartient au régime tropical humide caractérisée par deux saisons sèches (la grande saison sèche court de novembre à mi-mars et la petite saison sèche va de mi-juillet à mi-août) et deux saisons de pluies (la grande saison des pluies va de mi-mars à mi-juillet et la petite 
saison des pluies court de mi-août à fin octobre) (N'Guessan et al., 2014). L'évolution pluviométrique interannuelle est présentée à la figure 2 . La pluviométrie annuelle varie entre $857 \mathrm{~mm}$ et $1512 \mathrm{~mm}$ (1984-2015) avec une moyenne interannuelle de $1142 \mathrm{~mm}$.

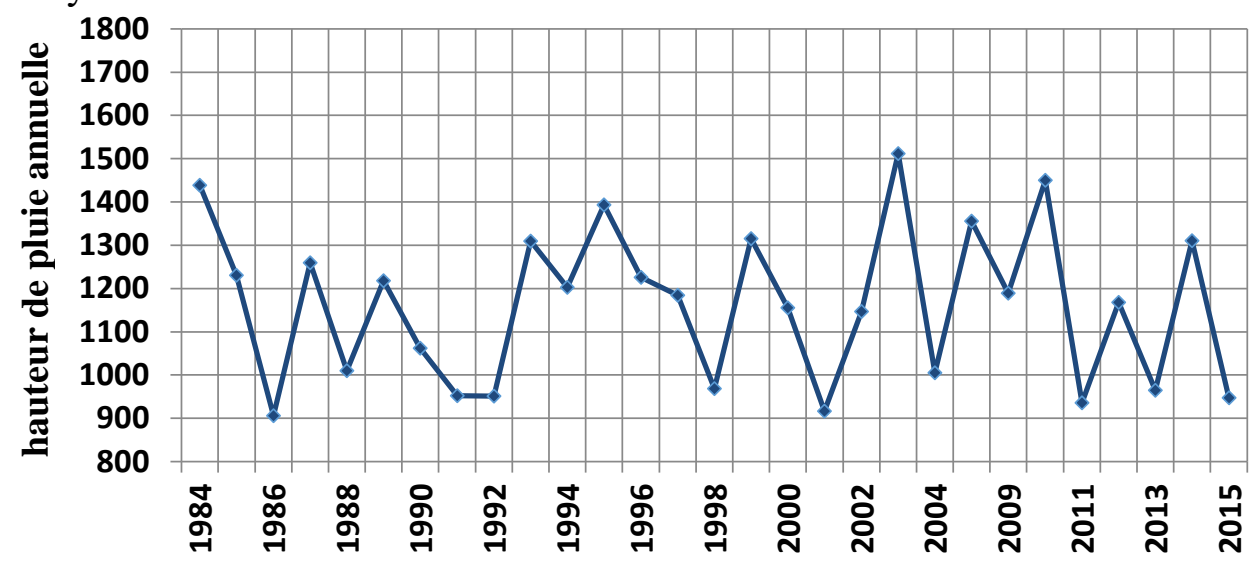

Figure 2 : Evolution interannuelle des pluies (mm)

\section{Méthodologie}

Etude de la dynamique spatio-temporelle de l'occupation du sol dans le bassin versant

Des images satellitaires Landsat TM (Thematic Mapper) du 16 Janvier 1986, ETM+ (Enhanced Thematic Mapper plus) du 20 Décembre 2000, OLI (Operational Land Imager) du 16 Janvier 2015 issues de la scène 197-055 et l'image Google Earth de Janvier 2016, ont été utilisées. Ces images ont toutes été acquises pendant la période sèche afin de réduire fortement leur nébulosité et limiter ainsi les biais atmosphériques (Hountondji, 2008).

L'étape de reconnaissance spectrale a été essentiellement visuelle (Bigot et al., 2005). Elle s'est faite grâce à l'image OLI et Google Earth. Cette reconnaissance visuelle a été confirmée ou infirmée par une visite de terrain et la prise de 30 échantillons par GPS (Global Positioning System). La campagne de terrain a permis de constater une organisation différente des couverts végétaux selon les compartiments géomorphologiques considérés.

L'amélioration du contraste des images a été faite comme indiqué dans l'étude de Irie et al., (2015). Plusieurs compositions colorées ont été réalisées dans le but d'associer les canaux qui véhiculent le maximum d'informations. Pour l'image de Janvier 1986, la composition colorée issue des canaux de base (Moyen infra-rouge, rouge et vert) a été retenue. Quant aux images de décembre 2000 et janvier 2015, la composition colorée composée des canaux de base (Rouge, Proche infra-rouge et Bleu) a été la plus discriminante.

L'étape de la classification a été possible grâce à l'algorithme du maximum de vraisemblance car elle a donné de bons résultats au cours des 
travaux de plusieurs auteurs (Bigot et al., 2005 ; Kouamé et al., 2007 ; Irié et al., 2015). Cette méthode repose sur les caractéristiques statistiques des échantillons représentatifs des classes identifiées sur l'image. L'algorithme affecte ensuite chaque pixel de l'image à la classe d'occupation du sol à laquelle il a la plus forte probabilité d'appartenir. La figure 3 résume la méthodologie adoptée.

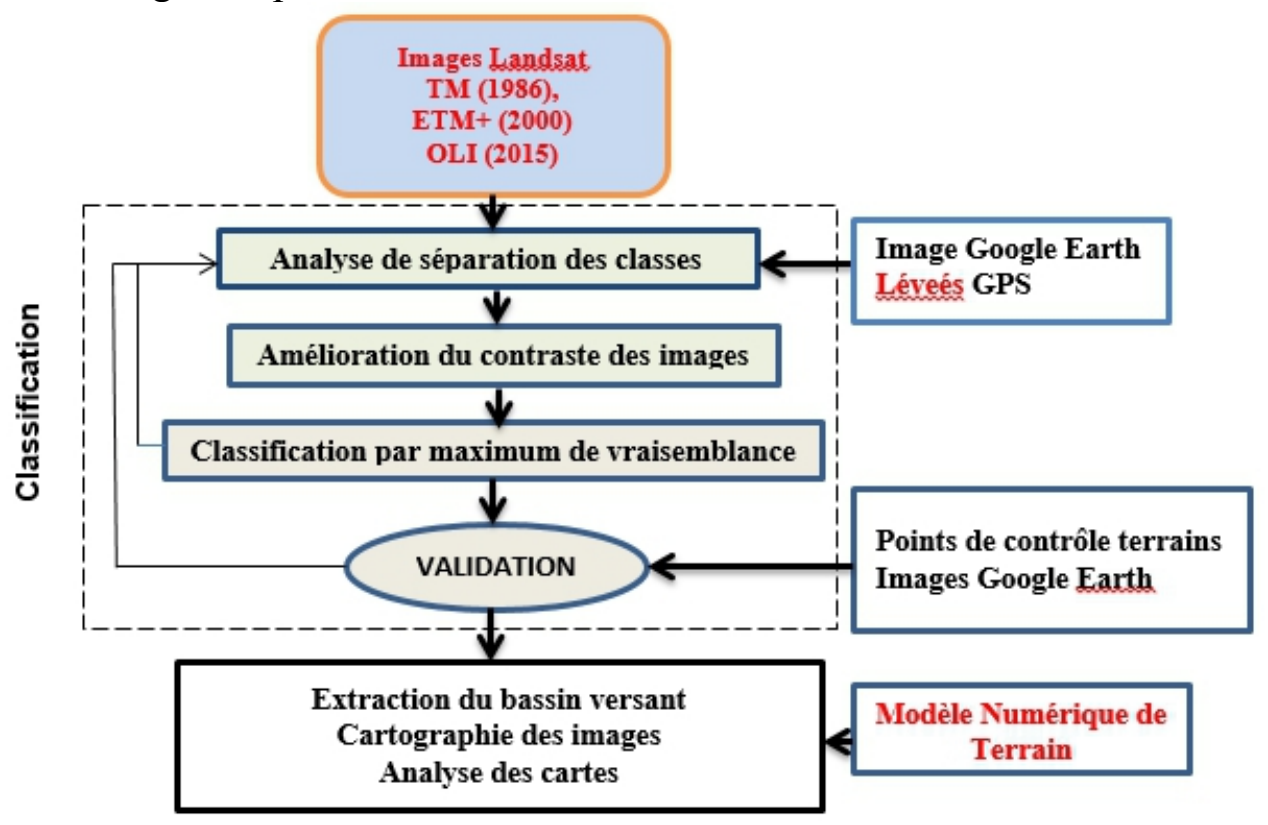

Figure 3 : Méthodologie de la cartographie de l'occupation du sol du bassin versant

L'extraction du bassin versant a été effectuée à partir d'image MNT ASTER de résolution $30 \mathrm{~m}$. Les limites obtenues du bassin versant ont permis de procéder à l'extraction proprement dit de l'espace d'étude sur les images satellitaires ainsi qu'à la détermination précise des surfaces afférentes à chaque classe d'occupation du sol dans le bassin versant.

\section{Caractérisation morphologique du bassin versant}

La caractérisation s'est faite à l'aide des cartes d'occupation du sol à différentes dates, de l'image MNT et des visites de terrain. La première étape de la caractérisation a été essentiellement visuelle. La présence d'ouvrages (Routes, ouvrages de franchissement) et de zones d'interruption probables de l'écoulement ont été détectées à travers les cartes de l'occupation du sol du bassin versant. Cette étape a été suivie d'une visite de terrain. La visite a permis de vérifier la position des ouvrages identifiés et le sens d'écoulement préférentiel des eaux lors d'averses pluvieuses. Les caractéristiques morphologiques du bassin versant déterminées à partir de la carte topographique de la zone d'étude à l'échelle $1 / 50000^{\text {ème }}$ ont été comparées à 
celles obtenues avec un Modèle Numérique de Terrain. Enfin ces caractéristiques morphologiques ont servi dans l'étude de l'impact de la dynamique de l'occupation du sol dans le bassin versant sur les apports en eau.

\section{Impact de la dynamique spatio-temporelle de l'occupation du sol dans le bassin versant sur ses apports en eau}

Le coefficient de ruissellement représente le rendement de la pluie (la fraction d'eau ruisselée jusqu'à l'exutoire par rapport à l'eau précipitée). Il est fonction de l'état de surface et de l'importance des précipitations. Dans le cadre de cette étude, nous analyserons les coefficients de ruissellements établies par certains auteurs, sur des bassins expérimentaux ayant certaines similarités avec la zone d'étude (Tableau 1).

Tableau 1 : Coefficient de ruissellement $(\mathrm{Kr})$ selon l'état de surface

\begin{tabular}{|c|c|c|c|}
\hline Etat de surface & Zone d'étude & $\mathrm{Kr}(\%)$ & Références \\
\hline \multirow{4}{*}{ Savane } & Toumodi (Côte d'Ivoire) & 15 & Dubreuil (1960) \\
\hline & Borotou (Côte d'Ivoire) & $12-16$ & Brou (1985) \\
\hline & Sud du Burkina Faso & 13 & $\begin{array}{l}\text { Fournier et al.,(2000) in } \\
\text { Fowe et al., 2012 }\end{array}$ \\
\hline & Nord du Burkina Faso & 17 & $\begin{array}{c}\text { Yacouba et al., (2002) in } \\
\text { Fowe et al., 2012 }\end{array}$ \\
\hline Forêt naturelle & Adiopodoumé (Côte d'Ivoire) & 7,8 & Roose (1977) \\
\hline \multirow{3}{*}{ Surface cultivée } & Adiopodoumé & 24 & Roose (1977) \\
\hline & Sud du Burkina Faso & 20 & $\begin{array}{l}\text { Fournier et al.,(2000) in } \\
\text { Fowe et al., 2012 }\end{array}$ \\
\hline & Nord du Burkina Faso & 24 & $\begin{array}{c}\text { Yacouba et al., (2002) in } \\
\text { Fowe et al., 2012 }\end{array}$ \\
\hline Jachère & $\begin{array}{l}\text { Adiopodoumé } \\
\text { Borortou }\end{array}$ & $\begin{array}{c}28 \\
22-42\end{array}$ & $\begin{array}{c}\text { Roose (1977) } \\
\text { Brou (1985) }\end{array}$ \\
\hline Plan d'eau & Sud et Nord du Burkina Faso & 100 & $\begin{array}{c}\text { Fournier et al.,(2000) et } \\
\text { Yacouba et al., (2002) in } \\
\text { Fowe et al., 2012 }\end{array}$ \\
\hline $\begin{array}{l}\text { Habitat à standing } \\
\text { (grand et moyen } \\
\text { standing) }\end{array}$ & Yopougon (Côte d'Ivoire) & $25-30$ & Bouvier et al.,(1987) \\
\hline Habitat populaire & Yopougon (Côte d'Ivoire) & $70-80$ & \\
\hline
\end{tabular}

Ensuite, à chaque état de surface de la zone d'étude a été attribué le coefficient de ruissellement approprié tenant compte de la connaissance approfondie de la zone d'étude. Ces valeurs sont valables pour une pente moyenne inférieure à $1 \%$.

Le coefficient de ruissellement moyen annuel du bassin versant est ensuite déterminé par pondération des surfaces des classes d'occupation du sol dans le bassin versant à partir de la formule 1:

$\mathbf{K}_{\mathbf{r}}=\frac{\sum K_{r i} A_{i}}{\sum A_{i}}$

$\mathbf{K}_{\mathbf{r i}}$ : le coefficient de ruissellement de l'état de surface i 
$\mathbf{A}_{\mathbf{i}}$ : Superficie occupée par la classe d'occupation du sol i dans le bassin versant

$\mathbf{K}_{\mathbf{r}}$ : le coefficient de ruissellement moyen annuel du bassin versant.

Pour l'étude de la dynamique temporelle de l'écoulement ( le calcul des lames d'eau ruisselées, des volumes ruisselés, des débits moyens et les débits de pointes à différentes dates), la méthode de Rodier-Auvray de l'ORSTOM-CIEH (1965) a été utilisée. Ce choix est lié d'une part à la disponibilité des données d'entrée de cette méthode, et d'autres part au domaine de validité de la méthode qui prend en compte les bassins versants de superficie inférieur à $200 \mathrm{~km}^{2}$. Egalement, la zone pluviométrique concernée par cette méthode, prend en compte la zone tropicale de transition dans laquelle la zone d'étude est située.

\section{Caractérisation des ruissellements amont et aval du dalot}

Une campagne de jaugeage a été effectué du 14/11/2017 au 20/11/2017 grâce à un moulinet de type OTT/C31 « 10001 » et un GPS de type Garmin etrex 10 à l'amont immédiat du dalot (6 $6^{\circ} 48.30^{\prime}$ de latitude Nord et $5^{\circ} 14.29^{\prime}$ de longitude Ouest) et à l'aval C'est-à-dire à l'entrée de la cuvette du barrage (6 ${ }^{\circ} 48.29^{\prime}$ de latitude Nord et $5^{\circ} 14.26^{\prime}$ de longitude Ouest). Ensuite, les vitesses moyennes ont été déterminées grâce à la méthode des sections moyennes et les débits amont et aval du dalot déduits afin de caractériser le ruissellement alimentant le barrage.

\section{Resultats et discussion}

\section{Validité de la classification des images satellitaires}

L'analyse par reconnaissance spectrale des images satellitaires a permis d'identifier cinq classes principales d'occupation du sol :

- La forêt (forêt galerie et îlot forestier),

- La savane (savane arborée et arbustive),

- Les cultures / jachères,

- L'habitat / sol nu,

- l'eau

La classification des différentes images a été évaluée à travers les matrices de confusion (Tableau 1, 2 et 3). Les précisions globales et les indices de Kappa ont été également utilisés pour la validation des images obtenues.

La classification de l'image de 1986 a donné une précision globale de $99 \%$ et un indice de kappa de 0,98. A travers la matrice de confusion, les précisions cartographiques sont comprises entre $96 \%$ et $100 \%$. 
Tableau 2 : Matrice de confusion de la classification de l'image Landsat de 1986

\begin{tabular}{|c|c|c|c|c|c|c|}
\hline & & \multicolumn{5}{|c|}{ pixels de vérification } \\
\hline & & Eau & $\begin{array}{c}\text { Habitat/ } \\
\text { sol nu }\end{array}$ & $\begin{array}{l}\text { Culture/ } \\
\text { Jachère }\end{array}$ & Savane & Forêt \\
\hline \multirow{6}{*}{$\begin{array}{l}\text { Pixels } \\
\text { affectés }\end{array}$} & Eau & 99,49 & 0 & 0 & 0 & 0 \\
\hline & Habitat/ sol nu & 0 & 100 & 0 & 0 & 0,52 \\
\hline & Culture/Jachère & 0,2 & 0 & $\mathbf{9 8 , 8 5}$ & 0,72 & 0,35 \\
\hline & Savane & 0 & 0 & 0,58 & 98,56 & 2,97 \\
\hline & Forêt & 0,31 & 0 & 0,58 & 0,72 & 96,15 \\
\hline & TOTAL & 100 & 100 & 100 & 100 & 100 \\
\hline
\end{tabular}

Précision globale : 99,01\%; Indice de Kappa : 0,98

L'image de 2000 a été classifiée avec une précision globale de $96 \%$ et un indice de kappa de 0,95 . Les précisions cartographiques sont comprises entre $94 \%$ et $99 \%$.

Tableau 3 : Matrice de confusion de la classification de l'image Landsat de 2000

\begin{tabular}{c|ccccccc} 
& & \multicolumn{5}{c}{ pixels de vérification } \\
\cline { 3 - 7 } \multicolumn{2}{c}{} & Eau & $\begin{array}{c}\text { Habitat/ } \\
\text { sol nu }\end{array}$ & $\begin{array}{c}\text { Culture/ } \\
\text { Jachère }\end{array}$ & Savane & Forêt \\
\hline \multirow{4}{*}{$\begin{array}{c}\text { Pixels } \\
\text { affectés }\end{array}$} & Eau & $\mathbf{9 9 , 5 7}$ & 0 & 0 & 0 & 0 \\
& Habitat/ sol nu & 0 & $\mathbf{9 6 , 6 3}$ & 0,19 & 0 & 0 \\
& Culture/Jachère & 0,26 & $\mathbf{3 , 3 7}$ & $\mathbf{9 4 , 4}$ & $\mathbf{3 , 3 8}$ & 1,61 \\
& Savane & 0 & 0 & $\mathbf{4 , 5 4}$ & $\mathbf{9 6 , 2 4}$ & 0 \\
& Forêt & 0,17 & 0 & 0,87 & $\mathbf{0 , 3 8}$ & $\mathbf{9 8 , 3 9}$ \\
& TOTAL & $\mathbf{1 0 0}$ & $\mathbf{1 0 0}$ & $\mathbf{1 0 0}$ & $\mathbf{1 0 0}$ & $\mathbf{1 0 0}$ \\
\hline
\end{tabular}

La classification de l'image de 2015 a donné une précision globale de $95 \%$ et un indice de kappa de 0.91 . Les précisions cartographiques sont comprises entre $91 \%$ et $99 \%$.

Tableau 4 : Matrice de confusion de la classification de l'image Landsat de 2015

\begin{tabular}{|c|c|c|c|c|c|c|}
\hline & \multirow{2}{*}{ 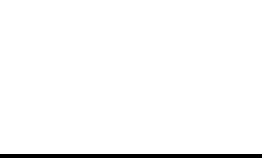 } & \multicolumn{5}{|c|}{ pixels de vérification } \\
\hline & & Eau & $\begin{array}{c}\text { Habitat/ } \\
\text { sol nu }\end{array}$ & $\begin{array}{l}\text { Culture/ } \\
\text { Jachère }\end{array}$ & Savane & Forêt \\
\hline \multirow{6}{*}{$\begin{array}{l}\text { Pixels } \\
\text { affectés }\end{array}$} & Eau & 99,07 & 0 & 0,02 & 0 & 0 \\
\hline & Habitat/ sol nu & 0 & 97,95 & 1,79 & 0 & 0 \\
\hline & Culture/Jachère & 0,41 & 2,02 & 91,42 & 0 & 5 \\
\hline & Savane & 0 & 0 & 0,27 & 96,82 & 3 \\
\hline & Forêt & 0,51 & 0,02 & 6,5 & 3,18 & 92 \\
\hline & TOTAL & 100 & 100 & 100 & 100 & 100 \\
\hline
\end{tabular}

Précision globale : 94,97\% ; Indice de Kappa : 0,91 
La classification des images a donné des précisions cartographiques et des précisions globales supérieures à $90 \%$ et des indices de Kappa supérieur à 0,90. Ces précisions cartographiques se trouvent dans les mêmes ordres de grandeurs que les valeurs obtenues par plusieurs auteurs, notamment, Kangah (2006), Sangne (2009), et Irié et al., (2015). Et d'après Pointus (2000) cité par Soro et al (2014), les résultats d'une classification d'images dont la valeur de Kappa est supérieure à 0,50 sont bons et exploitables. Ainsi, les présentes classifications qui ont permis de discriminer cinq classes d'occupation sont bonnes et exploitables.

\section{Analyse de la dynamique spatio-temporelle de l'occupation du sol du bassin versant}

L'analyse de la dynamique de l'occupation du sol s'est faite à travers l'étude des cartes de l'évolution de l'occupation du sol de 1986, 2000 et 2015 (Figure 4 - 6). Les modifications s'apprécient par les changements dans l'espace géographique et l'évolution des différentes surfaces (Tableau 5).

La carte de 1986 (figure 4) présente un bassin versant déjà affecté par les actions anthropiques, que sont l'urbanisation de la zone et le développement de zones de cultures. $31 \%$ et $34 \%$ de la surface du bassin sont occupé respectivement par l' habitat/sol nu et les cultures/jachères.
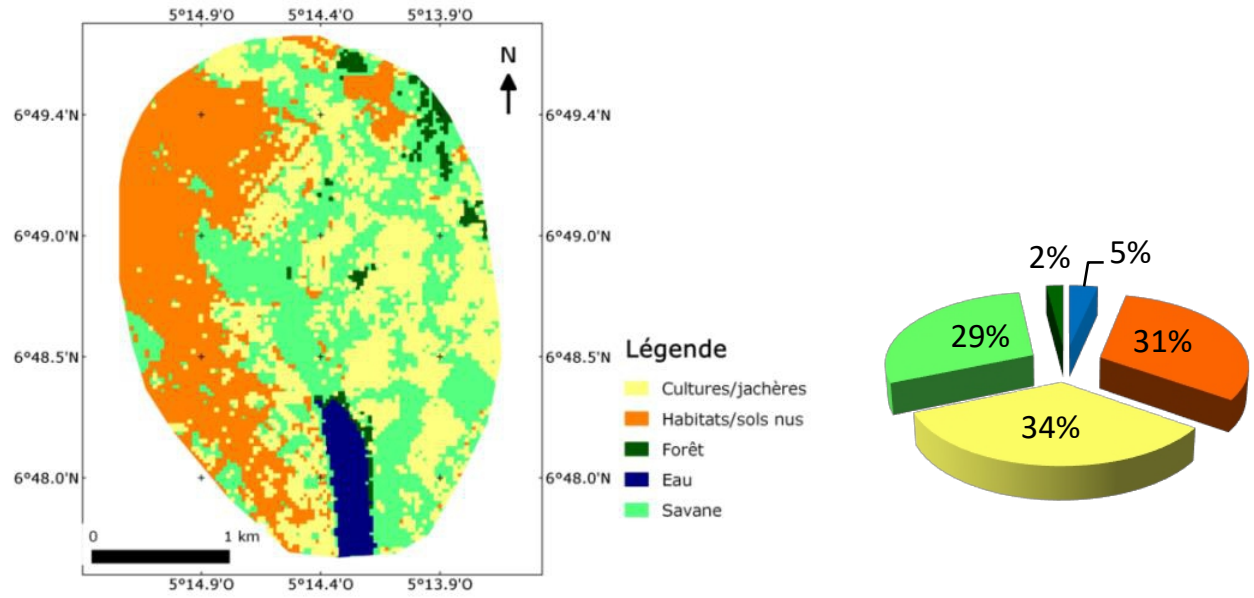

Figure 4 : Carte et taux d'évolution des classes d'occupation du sol de janvier 1986

La carte de 2000 (figure 5), présente un bassin versant sur lequel l'anthropisation a continué avec l'augmentation des surfaces occupées par les cultures/jachères et habitat/sol nu au détriment de la savane qui a considérablement diminué. $33 \%$ et $48 \%$ de la surface du bassin sont occupé respectivement par l'habitat/sol nus et les cultures/jachères. On assiste à une augmentation de la surface des forêts et une réduction notable de la surface du plan d'eau. 

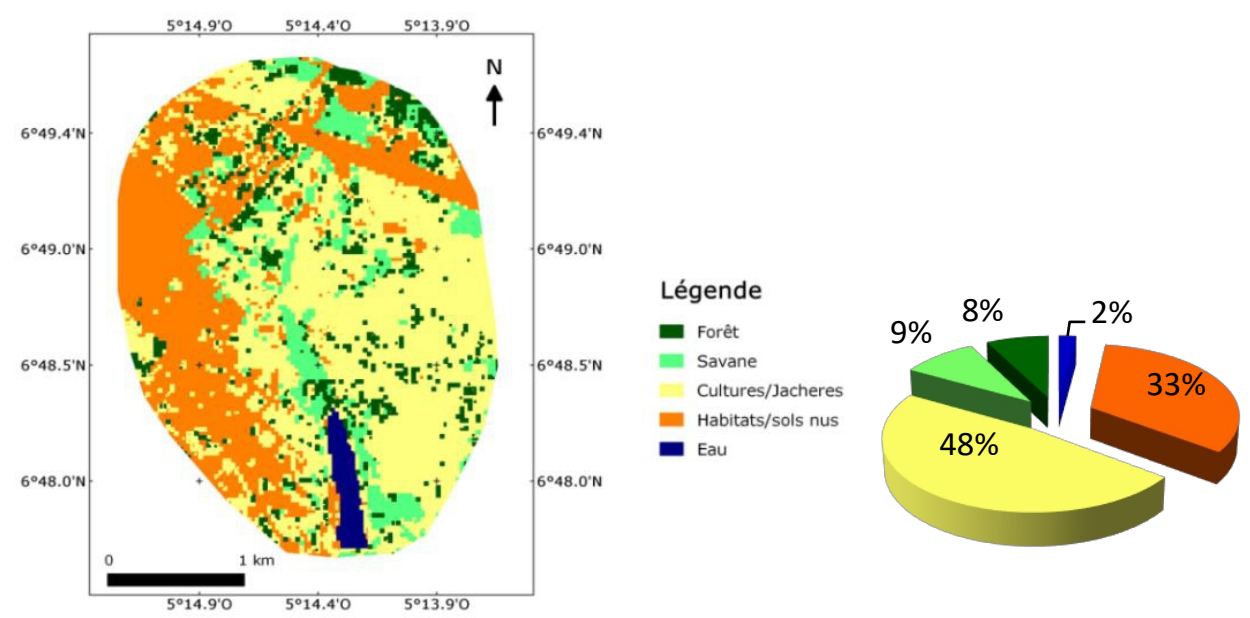

Figure 5 : Carte et taux d'évolution des classes d'occupation du sol de décembre 2000

L'image de 2015 (figure 6) présente un bassin versant complètement urbanisé avec 55\% de la surface du bassin versant occupée par l'habitat/sol nus au détriment des cultures/jachères et savane dont les surfaces ont diminué.
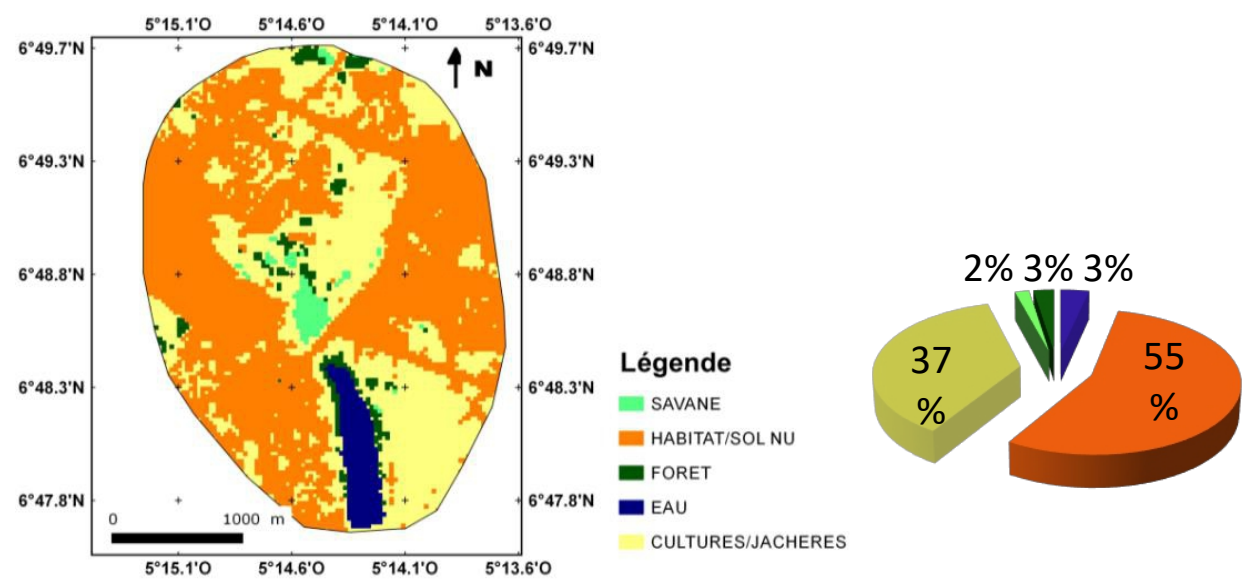

Figure 6 : Carte et taux d'évolution des classes d'occupation du sol de janvier 2015

En effet, en 1986, les classes, Habitat/sol nu, Cultures/Jachères et savane occupent des surfaces sensiblement égales (respectivement 258,3 hectares, 280,8 hectares et 242,04 hectares) (tableau 5).

De 1986 à 2000, la surface de la savane a diminué de 165,5 hectares en quatorze (14) ans soit une réduction annuelle de 11,8 hectares. Le fait notable en 2000 est l'augmentation de la surface des forêts de 46 hectares et la réduction du plan d'eau de 11,47 hectares. La réduction du plan d'eau est justifiée par la faible pluviométrie de l'année 2000 qui s'élevait à $1155,8 \mathrm{~mm}$ comparé à 1985 et 2014 respectivement arrosées par $1230 \mathrm{~mm}$ et $1310,9 \mathrm{~mm}$. La nette augmentation de la surface de la forêt en 2000 est probablement due 
à la régénérescence de certains espèces du fait de l'environnement général humide de la période 1993-2010. Par ailleurs, les zones de forêt constituées de forêt galerie, d'îlot forestier et d'espaces verts sont respectivement des zones hydromorphes, difficilement exploitables et des zones protégées par les autorités contre l'action humaine. Ce constat est similaire aux conclusions de Soro et al., (2014) dans l'étude de la dynamique de l'occupation du sol dans la région des lacs.

De 2000 à 2015, la classe habitat/sol nu a évoluée considérablement en augmentant de 186,31 hectares en quinze (15) ans, soit 12,4 hectares par an, au profit des classes Savane et Cultures/Jachères.

Tableau 5 : Evolution des surfaces des types d'occupation du sol

\begin{tabular}{cccc} 
& \multicolumn{3}{c}{ Surface (hectare) } \\
\cline { 2 - 4 } & Janvier 1986 & Décembre 2000 & Janvier 2015 \\
\hline Eau & 29 & 17,53 & 29,26 \\
Habitat/sol nu & $\mathbf{2 5 8 , 3}$ & $\mathbf{2 7 3 , 8 5}$ & $\mathbf{4 6 0 , 1 6}$ \\
Cultures/Jachères & $\mathbf{2 8 0 , 6}$ & $\mathbf{3 9 6 , 0 6}$ & $\mathbf{3 0 6 , 3 7}$ \\
Savane & 242,04 & 76,53 & 12,51 \\
Forêt & 17,19 & 63,22 & 18.89 \\
\hline
\end{tabular}

L'analyse de la dynamique spatio-temporelle de l'occupation du sol dans le bassin versant entre 1986 et 2015 a montré que l'urbanisation du bassin versant a débuté avant 1986 et s'est accélérée après 2000. Sylla (2002), dans le Nord de la Côte d'Ivoire et Eba (2013) dans le petit bassin versant de Gagnoa ont montré une importante transformation du paysage entre 1986 et 2000. Par ailleurs, cette accélération de l'urbanisation après 2000 pourrait s'expliquer par le déplacement massif de population vers Yamoussoukro, suite à la crise politico-militaire de 2002 et à la crise post-électorale de 2010 qu'a connu la Côte d'Ivoire. Selon le RGPH (2014), le taux d'urbanisation du département de Yamoussoukro est de $66.9 \%$. Or Thumerelle (1996) a révélé que l'explosion urbaine est liée à l'accroissement naturel et aux flux migratoires. En effet, les populations venues de divers horizons, en quête de nouvelles activités et d'autosuffisance alimentaire vont s'installer et contribuer à exploiter d'avantages les zones naturelles existantes. Les infrastructures ont favorisé l'implantation et l'augmentation des activités humaines, accentuant ainsi les modifications de l'occupation du sol et des états de surfaces (Mahé, 2006).

\section{Caractérisation morphologique du Bassin versant}

Le bassin versant du barrage de Nanan a connu différents étapes dans son évolution morphologique au cours du temps (figure 7 et 8). Entre 1986 et 2000, elle a subi une réduction de sa superficie avant d'être subdivisé en deux sous bassins versant après 2000 .

Avec les outils du SIG, la surface topographique du bassin versant en 
1986, a été estimée à $8,272 \mathrm{~km}^{2}$. Entre 1986 et 2000, le bassin versant topographique a subi une réduction de sa surface de $0,924 \mathrm{~km}^{2}$ et a désormais une surface de $7,348 \mathrm{~km}^{2}$.

Entre 2000 et 2015 le bassin versant a subi une seconde transformation. Il a été subdivisé en deux sous bassin versant séparés par une voie. Le sous bassin versant amont appelé sous bassin versant $\mathrm{N}^{\circ} 1$ (SBV1) avec une surface de 4,30 $\mathrm{km}^{2}$, draine le ruissellement amont qui traverse la voie de l'hôtel des députés à travers un dalot à double entrée $(2 \times 2,00 \times 2,00)$; Et le sous bassin versant aval appelé sous bassin versant $\mathrm{N}^{\circ} 2$ (SBV2) dont la surface est estimée à $3,048 \mathrm{~km}^{2}$ alimente directement le barrage.

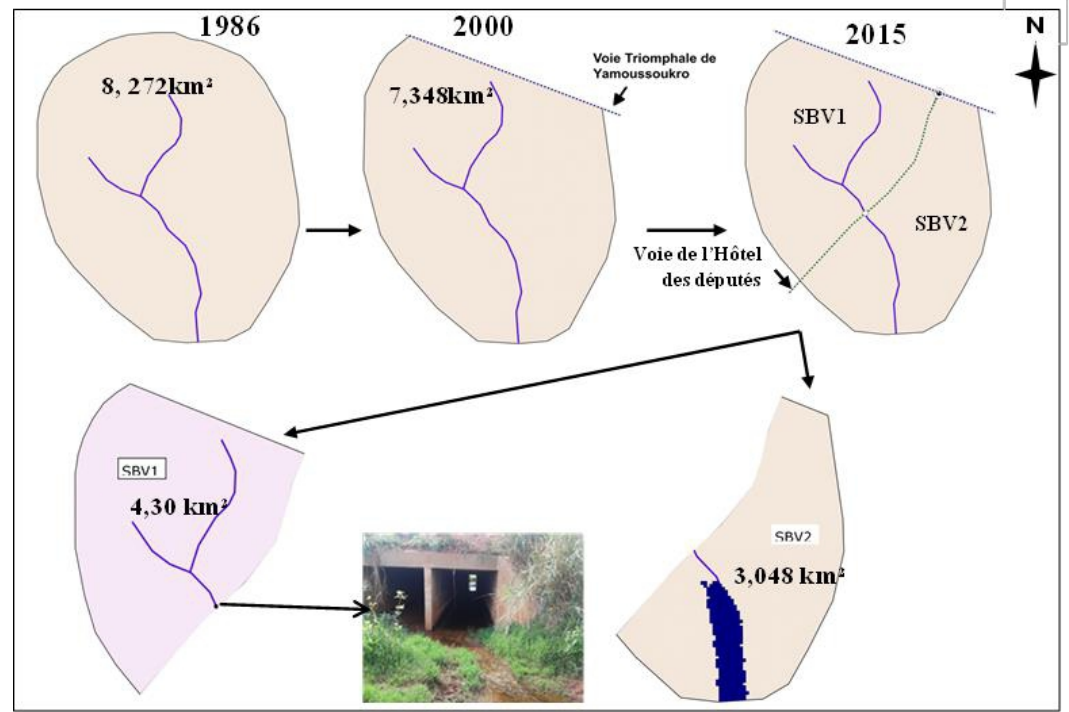

Figure 7 : Evolution morphologique du bassin versant et une vue aval du dalot

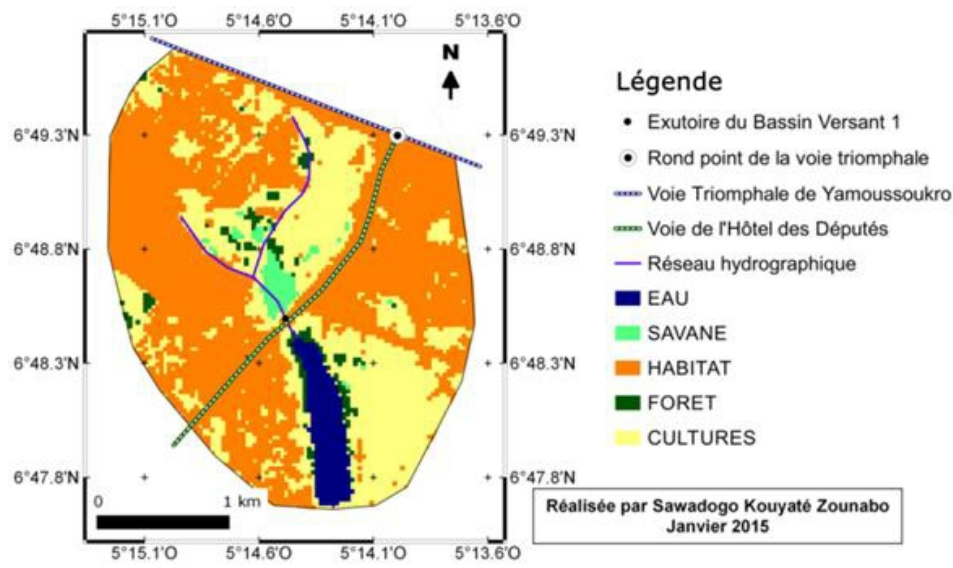

Figure 8 : Etat actuel de l'occupation du sol du bassin versant du barrage

Entre 1986 et 2000, la modification du bassin versant est justifiée par le démarrage des travaux de transfert de la capitale politique d'Abidjan à 
Yamoussoukro avec la réalisation de la voie Triomphale de Yamoussoukro en 1996. Ensuite, les travaux du transfert de la capitale politique entamés, ont continué après 2000 avec la construction de l'hôtel des députés ainsi que le démarrage des travaux de construction de la future assemblée Nationale, du futur Palais Présidentiel et d'infrastructures routières dont la voie de l'hôtel des députés. Ce sont autant d'action qui ont contribué à transformer la morphologie du bassin versant, dans lequel la plupart des édifices ont été réalisées.

Les caractéristiques morphologiques déterminées à partir du modèle

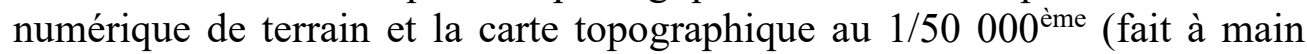
levée) sont comparées dans le tableau 6 :

Tableau 6 : Caractéristiques morphologiques du bassin versant

\begin{tabular}{cccccccc}
\cline { 2 - 7 } & $\begin{array}{c}\text { Superficie } \\
\left(\mathrm{km}^{2}\right)\end{array}$ & $\begin{array}{c}\text { Périmètre } \\
(\mathrm{Km})\end{array}$ & $\begin{array}{c}\text { Coefficient } \\
\text { de } \\
\text { compacité }\end{array}$ & $\begin{array}{c}\text { Altitude } \\
\text { maximale } \\
(\mathrm{m})\end{array}$ & $\begin{array}{c}\text { Pente } \\
\text { moyenne } \\
(\mathrm{m} / \mathrm{km})\end{array}$ & $\begin{array}{c}\text { Altitude } \\
\text { minimale } \\
(\mathrm{m})\end{array}$ & $\begin{array}{c}\text { Dénivelé } \\
\text { spécifique } \\
(\mathrm{m})\end{array}$ \\
\hline $\begin{array}{c}\text { Plan } \\
\text { topographique }\end{array}$ & 7,287 & 10,850 & 1,13 & 257 & 3,85 & 203 & 34,22 \\
\hline $\begin{array}{c}\text { MNT } \\
\text { ASTER 30m }\end{array}$ & 7,348 & 10,885 & 1,13 & 259 & 3,85 & 205 & 37,38 \\
\hline
\end{tabular}

Les caractéristiques morphologiques du bassin versant déterminées avec le MNT sont supérieures à celles déterminées avec une carte topographique au $1 / 50000^{\text {ème }}$. On observe un écart de $0.061 \mathrm{~km}^{2}$ et $0.035 \mathrm{~km}$ respectivement au niveau de la superficie et du périmètre du bassin versant. Un écart de $2 \mathrm{~m}$ est également observable au niveau des altitudes et de près de $3 \mathrm{~m}$ au niveau de la dénivelée spécifique. Les autres caractéristiques (pente moyenne et le coefficient de compacité) sont sensiblement identiques.

En effet, l'exploitation du MNT conduit à prendre en considération plus d'irrégularité (due à une donnée pixellaire) qu'un simple tracé à la main (Roche, 1963). Egalement, l'utilisation du MNT et de l'occupation du sol ont permis de tenir compte des barrières artificielles que sont les routes et les ouvrages comme préconisé par Roche (1963). Ce sont autant de raisons qui justifient les différences entre les caractéristiques morphologiques obtenues avec le MNT et celles obtenues avec la carte topographique délimité à main levée.

\section{Impact de la dynamique spatio- temporelle de l'occupation du sol du bassin versant sur les apports en eau Coefficient de ruissellement}

Pour les différentes classes d'occupation du sol, l'analyse de l'état de surface et des résultats d'études antérieures des coefficients de ruissellement de bassins versant expérimentaux ont donné les coefficients de ruissellement 
potentiels ci-dessous (tableau 7). La pente moyenne du bassin versant a été trouvée égale à $0.385 \%$ (tableau 6) qui est inférieure à $1 \%$. Cette valeur de pente a été retenue pour les différentes classes d'occupation du sol.

Tableau 7 : Coefficient de ruissellement selon l'état de surface

\begin{tabular}{cc}
\hline Etat de surface & Coefficient de ruissellement $\left(\mathrm{K}_{\mathrm{r}}\right)(\%)$ \\
\hline Eau & 100 \\
Habitat/sol nu & 50 \\
Cultures/Jachères & 24 \\
Savane & 15 \\
Forêt & 7.8 \\
\hline
\end{tabular}

Le coefficient de ruissellement retenu pour la savane est celui de Toumodi $\left(\mathrm{K}_{\mathrm{r}}=15 \%\right)$, qui se trouve dans la même zone climatique que Yamoussoukro. Le coefficient de ruissellement de l'eau est identique partout $\left(\mathrm{K}_{\mathrm{r}}=100\right)$. Quant au coefficient de ruissellement de la forêt celui d'Adiopodoumé $\left(\mathrm{K}_{\mathrm{r}}=7.8\right)$ a été utilisé car la forêt de la zone d'étude composée de forêt galerie et d'îlot forestier ont une densité importante du couvert végétal proche de la forêt naturelle. La valeur du coefficient de ruissellement des zones de cultures, au Sud et Nord de la Côte d'Ivoire comme au Burkina Faso sont les mêmes. C'est ainsi que cette valeur $\left(\mathrm{K}_{\mathrm{r}}=24 \%\right)$ a été retenu. Concernant le coefficient de ruissellement de l'habitat / sol nu, nous avons considéré le fait que la zone d'étude est composé d'habitat populaire d'une part et de quartier haut standing d'autre part la moyenne des deux coefficients a été retenue $\left(\mathrm{K}_{\mathrm{r}}=50\right)$.

Le coefficient de ruissellement moyen annuel calculé à partir des coefficients de ruissellement obtenus sur des bassins versant expérimentaux pour différentes dates est présenté à la figure 9.

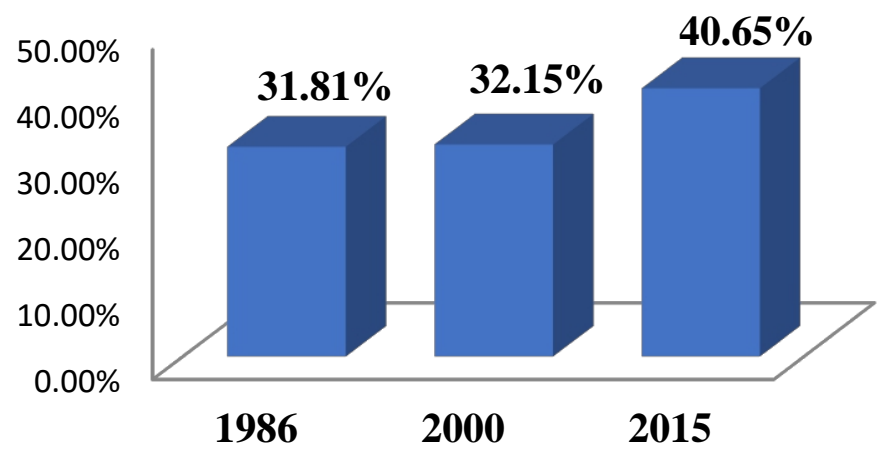

Figure 9 : Evolution temporelle du coefficient de ruissellement moyen annuel

Le coefficient de ruissellement moyen est resté constant entre1986 et 2000 pourtant, l'état de surface a continué d'être affecté par les actions anthropiques. Cela pourra se justifier par le fait que les surfaces de savanes 
perdues en 2000, ont été compensées par des surfaces de Cultures/jachères et la régénérescence de la forêt dont le couvert végétal retient selon sa densité, sa nature et l'importance de la précipitation une proportion de l'eau atmosphérique.

En 2015, on assiste à une augmentation du coefficient de ruissellement qui passe de $32,15 \%$ à 40,65\%. En effet, les modifications entre 2000 et 2015 sont exclusivement dû à l'urbanisation. Or l'extension et la densification du tissu urbain provoquent une incontestable augmentation des coefficients de ruissellement des bassins versant (Fehri et al., 2016). L'urbanisation se présente donc comme le facteur anthropique à la base de l'augmentation du coefficient de ruissellement moyen sur le bassin versant étudié. Egalement, l'augmentation de la valeur du coefficient de ruissellement moyen met en évidence l'augmentation des surfaces imperméabilisées et des quantités d'eau ruisselée.

\section{la dynamique temporelle de l'écoulement dans le bassin versant}

L'évolution temporelle des apports en eau et des débits sont présentés dans tableau 8 ci-dessous :

Tableau 8: Evolution temporelle des apports en eau du bassin versant

\begin{tabular}{lllll} 
& $\mathbf{1 9 8 6}$ & $\mathbf{2 0 0 0}$ & $\mathbf{2 0 1 5}$ & \\
\cline { 4 - 5 } & & & SBV1 & SBV2 \\
\hline Surface du bassin versant $\left(\mathbf{k m}^{\mathbf{2}}\right)$ & 8,273 & 7,348 & 4,30 & 3,048 \\
Lame d'eau ruisselée $(\mathbf{m m})$ & $\mathbf{4 1 , 7 0}$ & $\mathbf{4 3 , 3 0}$ & $\mathbf{4 5 , 4 0}$ & $\mathbf{4 6 , 4 0}$ \\
Volume ruisselé $\left(\mathbf{m}^{\mathbf{3}}\right)$ & 344984,10 & 318152,20 & 195220 & 141427,20 \\
Débit moyen $\left(\mathbf{m}^{\mathbf{3}} / \mathbf{s}\right)$ & 9,5 & 10 & 7,20 & 6,05 \\
Débit décennal $\left(\mathbf{m}^{\mathbf{3}} / \mathbf{s}\right)$ & 23,75 & 25 & 18,1 & 15,1 \\
\hline
\end{tabular}

La lame d'eau ruisselée a augmenté de 1986 à 2015 en passant de 41,7 $\mathrm{mm}$ à 45, $4 \mathrm{~mm}$ et 46,40 $\mathrm{mm}$. Le volume ruisselé a diminué de 1986 à 2000 de $26832 \mathrm{~m}^{3}$, suite à la réduction de la surface du bassin versant entre 1986 et 2000. Le débit moyen et le débit décennal ont également augmenté au cours du temps. L'augmentation de la fraction urbaine du bassin versant ayant abouti au morcellement de celui-ci expliquent le mieux les résultats obtenus. Car, les impacts de l'urbanisation touchent autant la quantité moyenne d'eau écoulée que la dynamique temporelle du débit (Bahar, 2015).

\section{Caractérisation du ruissellement amont et aval du dalot}

Les vitesses moyennes (figure 10) et les débits (figure 11) à l'amont et à l'aval du dalot obtenus sont présentés ci-dessous.

Les vitesses moyennes à l'aval du dalot sont très inférieures à ceux de l'amont du dalot. Le 20/11/2017, la vitesse moyenne du ruissellement est égale 
$0.20 \mathrm{~m} / \mathrm{s}$ à l'amont alors qu'à l'aval elle ne représente que $0.15 \mathrm{~m} / \mathrm{s}$, soit $3 / 4$ de la vitesse amont.

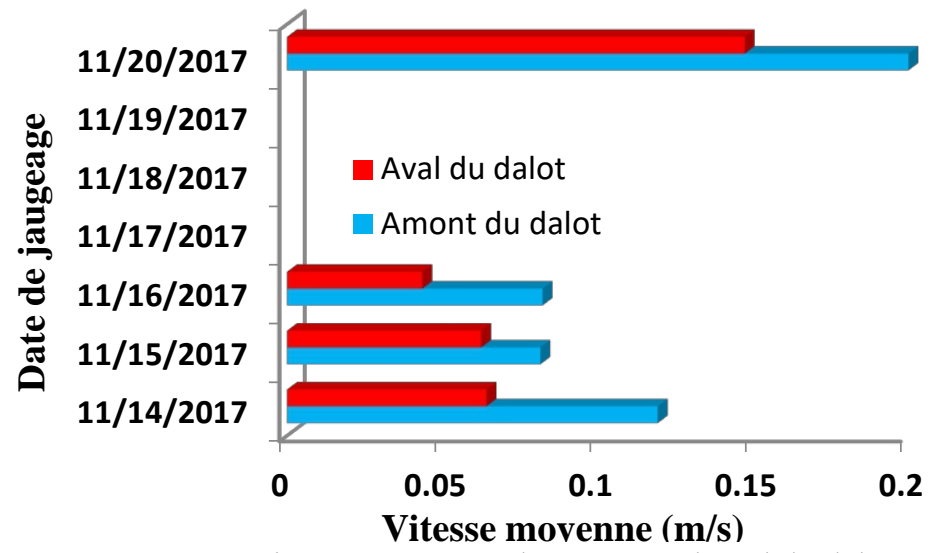

Figure 10 : Vitesse moyenne à l'amont et à l'aval du dalot

Les débits à l'aval du dalot sont très inférieurs aux débits à l'amont du dalot. Le 20/11/2017, un ruissellement amont née d'un événement pluvieux, de débit 20,8 1/s en traversant le dalot pour alimenter le barrage, se retrouve avec un débit aval de 14,70 1/s à l'entrée de la cuvette du barrage. Soit, plus de $30 \%$ du débit amont perdu.

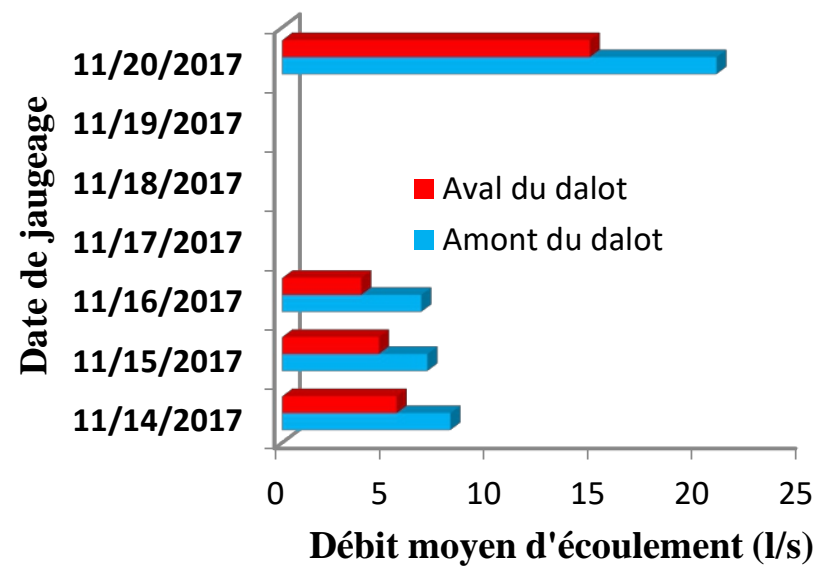

Figure 11 : Débit moyen d'écoulement à l'amont et à l'aval du dalot 
La figure 12 présente l'état du ruissellement amont et aval du dalot. La photo (a) présente un ruissellement naturel à l'amont, entrant dans le dalot par contre la photo (b) présente une sortie aval du dalot avec une stagnation visible au pied de celui-ci. Les photos (a) et (b) ont été prises le même jour.

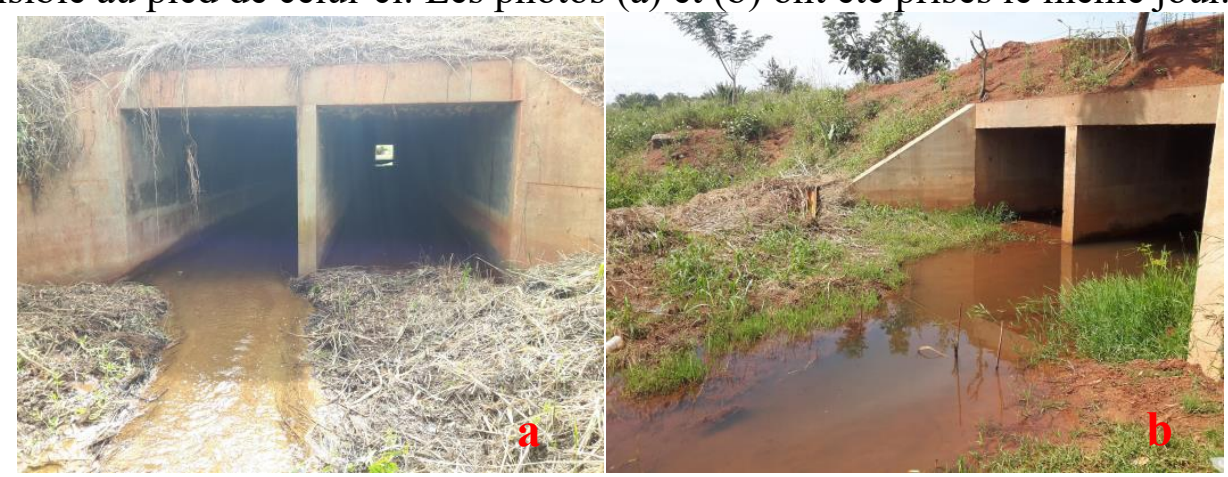

Figure 12: Ruissellement à l'amont (a) et Ruissellement à l'aval du dalot (b)

La figure 13 montre l'évolution temporelle du ruissellement aval en deux périodes (2016 et 2017). Sur la photo (c), le ruissellement se fait naturellement à travers un chenal, et la photo (d) présente un ruissellement diffus, stagnant.
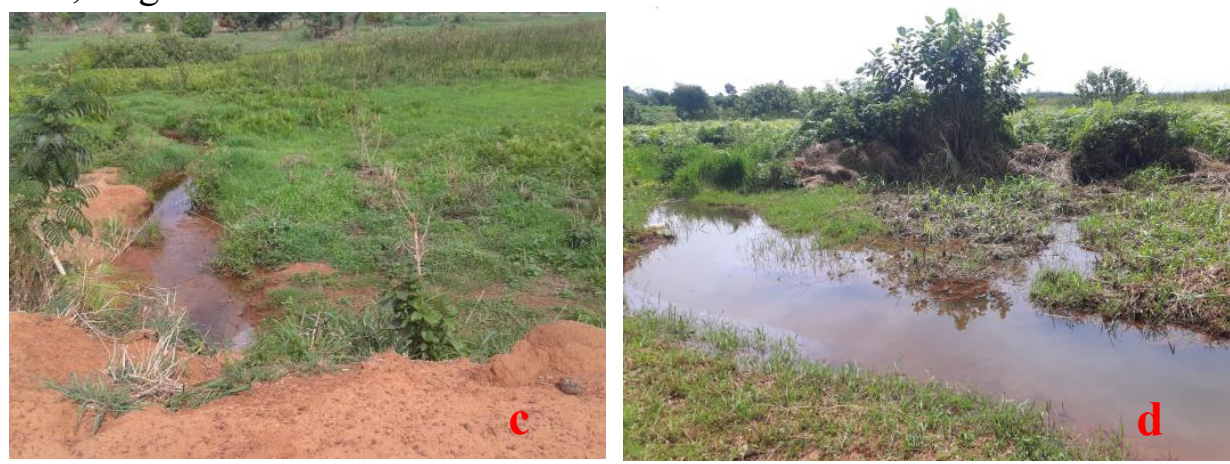

Figure 13 : Evolution temporelle du ruissellement à l'aval du dalot; (c) photo du 30/03/2016; (d) photo du 20/11/2017

L'estimation de la vitesse moyenne et du débit à l'amont et à l'aval du dalot et l'analyse des photos relatives à l'état du ruissellement à ces endroits ont montré une différence significative entre l'écoulement à l'amont et l'aval du dalot. Le débit du ruissellement aval ne représente environ que les $2 / 3 \mathrm{du}$ débit amont. En effet, les amas de terre restés après les travaux de réalisation de la voie de l'hôtel des députés et provenant de l'érosion du talus ont été enherbés au fur à mesure et constitue aujourd'hui un obstacle à l'écoulement. Ce qui a entrainé un ralentissement du ruissellement et une stagnation de l'eau à l'aval du dalot. Cette stagnation a favorisé l'infiltration d'où l'alimentation de la nappe souterraine au détriment de la retenue. Ainsi, la retenue subit une réduction de ces apports du fait des transferts d'eau inter-bassins et de 
l'enherbement. Cela pourrait justifier la réduction du niveau du plan d'eau de la retenue. Pourtant, la mise en valeur des parcelles par les riziculteurs est étroitement liée à l'appréciation de la quantité d'eau disponible dans la retenue. Cette appréciation se faisant de façon visuelle. Une observation en baisse de la quantité d'eau peut être un facteur de découragement et d'abandon des parcelles.

\section{Conclusion}

Les cartes réalisées à partir des images satellites, bien que de faible résolution spatiale, ont permis de dégager la tendance générale de l'occupation des sols sur 30 ans. Elle révèle une urbanisation accélérée du bassin versant avec l'augmentation des surfaces habitats/des sols nus aux dépens des savanes et surfaces agricoles.

Egalement, les outils du SIG ont montré leur efficacité dans l'étude et la compréhension des transformations survenues sur le bassin versant étudié. Avec une surface initiale de $8,272 \mathrm{~km}^{2}$, le bassin versant de Nanan a connu divers transformations suite aux travaux de construction de la future zone administrative de Yamoussoukro.

De ce qui précède, il ressort que suite à l'urbanisation accélérée, le bassin versant a subi plusieurs transformations morphologiques qui ont agi sur son fonctionnement hydrologique et diminué ces apports en eau vers la retenue compromettant la durabilité des activités rizicoles. Il serait nécessaire pour redonner des conditions favorables d'écoulement, de procéder à un désherbage et un curage du chenal entre l'aval du dalot et la cuvette de la retenue. Egalement, pour éviter cet état de fait, un projet d'aménagement d'ouvrages routiers à l'amont immédiat d'une retenue doit intégrer les caractéristiques de celui-ci au projet.

\section{References:}

1. Ardoin B.S., Lubes-Niel H., Servat E., Dezetter A., Boyer J.F. (2003) : Analyse de la persistance de la sècheresse en Afrique de l'Ouest: Caractérisation de la situation de la décennie 1990. IAHS Publication, Vol.278, 223-228.

2. Bahar S. (2015) : Impact de l'urbanisation sur la réponse hydrologique des bassins versants urbains, Thèse de doctorat de l'Université Pierre et Marie Curie, 186p.

3. Bigot S., Brou Y.T., Oszwald J., Diedhou A (2005) : Facteurs de la variabilité pluviométrique en Côte d'Ivoire et relations avec certaines modifications environnementales. Sécheresse, Vol.16 (1), 5-13.

4. BNETD (Bureau National d'Etudes Techniques et de Développement). (1997): Plan directeur d'urbanisme de la ville de Yamoussoukro. Rapport technique, 90p. 
5. Bouvier C., Berthelot M., Janeau J. L. (1987): Campagne de simulation de pluie en milieu urbain, Yopougon.. ORSTOM-CIEH, 16p.

6. Brou Kouamé (1985): Jaugeages sur grandes rivières à Borotou, simulation de la pluie, rapport de stage, laboratoire d'hydrologie, ORSTOM, Centre d'Adiopodoumé (Côte d'Ivoire), 44p.

7. Dubreuil P. (1960) : Etude hydrologique de petits bassins versants en Côte d'Ivoire, Rapport Général, zone de savane, 169p.

8. Eba A. E. L. (2013) : Approche géomatique pour la délimitation des périmètres de protection autour des ressources en eau de surface destinées à la consommation : cas de la retenue d'eau de Gagnoa (centre-ouest de la Côte d'Ivoire). Thèse de l'Université Félix Houphouët Boigny.

9. Ferhi N. et Yadh Z. (2016) : Étude de l'impact de l'extension et de la densification du tissu urbain sur les coefficients de ruissellement dans le bassin versant des oueds El-Ghrich et El-Greb (Tunis) par l'application de la méthode SCS aux évènements de septembre 2003 », Physio-Géo,Volume 10, URL : http://physio-geo.revues.org/4769 ; DOI : $10.4000 /$ physio-geo.4769

10. Fowe T., Karambiri H., Hamma Y., Barbier B., Paturel J. E. et Ibrahim B.(2012) : Impacts des scenarii climatiques et de l'occupation des sols sur la ressources en eau du bassin versant du Nakambé (BF), Climat et Developpement, $\mathrm{N}^{\circ} 14,15 \mathrm{p}$.

11. Fowe T., Karambiri H., Paturel J.-E., Poussin J.-C., et Cecchi P., (2015) : Water balance of small reservoirs in the Volta basin: A case study of Boura reservoir in Burkina Faso. Agricultural Water Management, Vol. 152, 99-109.

12. Goula B.T.A., Savane I., Konan B., Fadika V., Kouadio G.B. (2006) : Impact de la variabilité climatique sur les ressources hydriques des bassins de N'zo et N'zi en Côte d'Ivoire (Afrique tropicale humide).Vertigo, Vol. 1,1-12.

13. Hountondji I.C.H., (2008) : Dynamiques environnementales en zones sahélienne et soudanienne de l'Afrique de l'Ouest: Analyse des modifications et évaluation de la dégradation du couvert végétal. Thèse pour obtenir le grade de Docteur en Sciences de l'université de Liège, Belgique, 153p,.

14. I. Braud (2011): Pourquoi et comment étudier l'hydrologie des bassins versants périurbains. 1ere conférence thématique de l'OTHU, Jun 2011, Villeurbanne, France. GRAIE, p. 5 - p. 16,

15. Irie G. R., Soro G. E., Goula B. T. A. (2015) : Changements d'états de surface et évolutions spatio-temporelles des précipitations sur le bassin versant de la Marahoué (Côte d'Ivoire), International Journal of Innovation and Applied Studies ISSN 2028-9324 Vol. 13 No. 2 Oct. 
2015, pp. 386-397 Innovative Space of Scientific Research Journals http://www.ijias.issr-journals.org/.

16. J. Rodier et C. Auvray, (1965) : Estimation des débits de crues décennales des bassins versants de superficie inférieure à $200 \mathrm{~km}^{2}$ en Afrique Occidentale, ORSTOM-CIEH, 48p

17. Kangah A., 2006 : Utilisation de la télédétection et d'un système d'information géographique (SIG) pour l'étude des pressions anthropiques sur les paysages géomorphologiques des savanes subsoudanaises : exemple du degré carré de Katiola (cendre-nord Ivoirien), Thèse, Université de Cocody-IGT, 199p.

18. K.E.Kouakou, B.T.A. Goula, I. Savane (2007) : Impact de la variabilité climatique sur les ressources en eau de surface en zone tropicale humide : Cas du bassin versant transfrontalier de la Comoé (Côte d'Ivoire-Burkina Faso), European Journal of Scientific Research, 16(1), pp.31-43,

19. Kouame K.F., Bernier M., Gone D.L., Saley M.B., Lefebvre R., Soro N., Koudou A., (2007) : Intégration de données géospatiales dans un modèle hydrologique distribué pour la simulation des écoulements des eaux en milieu tropical humide de Côte d'Ivoire (Afrique de l'Ouest). Revue de télédétection, 7(1-2-3-4) : 217-235.

20. Kouassi A. M., (2007) : Caractérisation d'une modification éventuelle de la relation pluie-débit et ses impacts sur les ressources en eau en Afrique de l'Ouest : cas du bassin versant du N'zi (Bandama) en Côte d'Ivoire. Thèse de Doctorat unique, Université de Cocody, $210 \mathrm{p}$,

21. Mahé G. (2006) : Variabilité pluie-débit en Afrique de l'Ouest et Centrale au $20^{\text {ème }}$ siècle : Changements hydro-climatiques, occupation du sol et modélisation hydrologique.

22. N'Guessan K. A., Kouassi A. M., Gnaboa R, Traoré K.S., Houenou P.V (2014) : Analyse de phénomènes hydrologiques dans un bassin versant Urbanisé : Cas de la ville de Yamoussoukro (Centre de la Côte d'Ivoire), Larhyss Journal, ISSN 1112-3680, n¹7, Mars 2014, pp. 135-154,.

23. P. Cecchi, F.Gourdin, S.Kone, D. Corbin, E.Jackie, A. Casenave (2007) : Les petits barrages du Nord de la Côte d'Ivoire : Inventaire et potentialités hydrologiques. Sécheresse, 20(1), pp.11222.

24. Projet Riz Centre (1998): Rapport technique de réhabilitation du Périmètre irrigué de Nanan, $45 \mathrm{p}$,

25. RGPH, (2014) : Recensement Général de la Population et de l'Habitat 2014, Résultats globaux, Secrétariat technique permanent du comité technique du RGPH, 26p,. 
26. Roche M. (1963): Hydrologie de surface, Gauthier-Villars et ORSTOM, Paris, France, 429p.

27. Roose E. (1977) : Erosion et ruissellement en Afrique de l'ouest, vingt années de mesures en petites parcelles expérimentales, Travaux et documents de l'ORSTOM, N78, 107p, ORSTOM paris

28. Sangne Y. C. (2009) : Dynamique du couvert forestier d'une aire protégée soumise aux pressions anthropique : cas de la forêt classée de Téné dans le département d'Oumé (Centre-ouest de la Côte d'Ivoire). Thèse Unique. Université de Cocody. Abidjan (Côte d'Ivoire), 220 p.

29. Soro T.D., Soro N., Oga Y.M-S., Lasm T., SORO G., Ahoussi K.E., Biemi J. (2011) : La variabilité climatique et son impact sur les ressources en eau dans le degré carré de Grand-Lahou (Sud-Ouest de la cote d'Ivoire), Géographie physique et Environnement, Vol. 5, http://physiogeo.revues.org/1581; DOI : 10.4000/physio-geo.1581.

30. Soro, G., Ahoussi, E. K., Kouadio, E. K., Soro, T. D., Oulare, S., Saley, M. B., \& Soro, N. (2014) : La dynamique de l'occupation du sol dans la région des Lacs ( Centre de la Côte d'Ivoire ), 10(3), 146-160,

31. Sylla D. (2002) : Dynamique de l'occupation du sol dans le Nord de la Côte d'Ivoire (1986-2000): l'exemple du département de Korhogo. DEA, Université de Cocody-Abidjan, Côte d'Ivoire, 67p.

32. Thumerelle J., (1996) : Peuples en mouvement. La mobilité spatiale des populations. Dossier des images économiques du monde, Sedes, $326 \mathrm{p}$.

33. Yao A.B., Goula B.T.A., Kouadio Z.A., Kouakou K.E., Kante A.et Sambou S. (2012) : Analyse de la variabilité climatique et quantification des ressources en eau en zone tropicale humide : cas du bassin versant de la Lobo au Centre-Ouest de la Cote d'Ivoire, Revue Ivoirienne des Sciences et Technologie, 19, 136-157. 